\title{
EFECTO DE DOS MANIOBRAS MANUALES CÍCLICAS DE RECLUTAMIENTO ALVEOLAR SOBRE GASES SANGUÍNEOS EN PERROS ANESTESIADOS
}

\section{EFFECT OF TWO MANUAL CYCLICAL ALVEOLAR RECRUITMENT MANEUVERS ON THE VALUE OF BLOOD GASES IN ANESTHETIZED DOGS}

\author{
Edwin Buriticá Gaviria ${ }^{1}$, Diego Echeverry Bonilla², Diego Ospina Argüelles ${ }^{3}$
}

\begin{abstract}
${ }^{1}$ Médico Veterinario Zootecnista, Especialista en docencia universitaria, Especialista Clínico en pequeñas especies animales, M.Sc. en Ciencias Veterinarias. Grupo de Investigación en Medicina y Cirugía de Pequeños Animales. Universidad del Tolima, Código postal 730006299, Ibagué-Tolima, Colombia, e-mail: efburiticag@ut.edu.co; ${ }^{2}$ Médico Veterinario Zootecnista, Especialista en docencia universitaria, M.Sc. en técnicas de diagnóstico animal, Ph.D. en Veterinaria. Líder del Grupo de Investigación en Medicina y Cirugía de Pequeños Animales. Ibagué-Tolima, Colombia, e-mail: decheverry@ut.edu.co; ${ }^{3}$ Médico Veterinario Zootecnista, Especialista en pedagogía. Grupo de Investigación en Medicina y Cirugía de Pequeños Animales. Ibagué-Tolima, Colombia, e-mail: daospina@ut.edu.co
\end{abstract}

Rev. U.D.C.A Act. \& Div. Cient. 19(1): 161-168, Enero-Junio, 2016

\section{RESUMEN}

Las atelectasias durante la realización de procedimientos anestésicos afecta el intercambio gaseoso en seres humanos y en animales. Esta condición, se puede corregir, empleando maniobras de reclutamiento alveolar. El presente estudio evaluó el efecto de dos protocolos cíclicos de reclutamiento alveolar, mediante el incremento de la presión tele-espiratoria (PEEP), sobre seis parámetros de gases arteriales, en 28 caninos anestesiados. Los animales fueron asignados aleatoriamente en dos grupos, según la maniobra de reclutamiento alveolar (MRA) a realizar; grupo 1: Maniobra 1 (M1), reclutado con PEEP de $10 \mathrm{cmH}_{2} \mathrm{O}$ y grupo 2: Maniobra 2 (M2), reclutado con PEEP de $20 \mathrm{cmH}_{2} \mathrm{O}$. Las MRA, se realizaron empleando una unidad de respiración artificial manual (AMBU). Para cada maniobra, se evaluó la $\mathrm{PaO}_{2}$, $\mathrm{PaCO}_{2}, \mathrm{TCO}_{2}, \mathrm{PaO}_{2} / \mathrm{FiO}_{2}, \mathrm{SpO}_{2}$ y $\mathrm{CaO}_{2}$, en cuatro tiempos a saber: T1 (basal en preanestesia), T2 (pre-reclutamiento), T3 (30 min post-maniobra) y T4 (24 h post-maniobra). En el tiempo T2, los valores de $\mathrm{PaO}_{2}, \mathrm{PaCO}_{2}, \mathrm{TCO}_{2}, \mathrm{SpO}_{2}$ y $\mathrm{CaO}_{2}$ presentaron un incremento significativo $(\mathrm{p}<0,05)$, respecto al tiempo basal T1. No se evidenciaron cambios estadísticos significativos tras realizar cada una de las MRA en el tiempo T3; sin embargo, hubo un incremento significativo $(\mathrm{p}<0,05)$ en la $\mathrm{PaO}_{2}$, de $13,5 \%$ y la $\mathrm{PaO}_{2} / \mathrm{FiO}_{2}$, del $13,5 \%$, en el tiempo T4, tras realizar la maniobra M2. Se concluye, que la realización de MRA, mediante el empleo de $A M B U$, es una alternativa económica y segura de ventilación asistida, tras su empleo con PEEP inferiores a $20 \mathrm{~cm}_{2} \mathrm{O}$, en animales clínicamente sanos.
Palabras clave: Anestesia, atelectasia, gasometría, oxigenación, ventilación manual.

\section{SUMMARY}

The atelectasis during anesthesia affects gas exchange in humans and animals; this can be corrected using alveolar recruitment maneuvers (MRA). This study evaluated the effect of two cyclic alveolar recruitment protocols by increasing the positive end expiratory pressure (PEEP) on six parameters of arterial blood gases in 28 anesthetized bitches. The animals were randomly distributed into two groups according to the alveolar recruitment maneuver (MRA) to perform; Group 1: Maneuver 1 (M1) recruited with PEEP of $10 \mathrm{cmH} 2 \mathrm{O}$ and group 2: Maneuver 2 (M2) with PEEP $20 \mathrm{cmH} 2 \mathrm{O}$ recruited with $20 \mathrm{cmH}_{2} \mathrm{O}$ PEEP. Cyclic maneuvers were performed using ambulatory manual breathing units (AMBU). The $\mathrm{PaO}_{2}$, $\mathrm{PaCO}_{2}, \mathrm{TCO}_{2}, \mathrm{PaO}_{2} / \mathrm{FiO}_{2}, \mathrm{SpO}_{2}$ y $\mathrm{CaO}_{2}$ values was analyzed for each MRA in four times; T1 (baseline in preanesthesia), T2 (before of the recruitment), T3 (30 minutes after maneuver) and T4 (24 hours after maneuver). In the T2 moment the $\mathrm{PaO}_{2}, \mathrm{PaCO}_{2}, \mathrm{TCO}_{2}, \mathrm{SpO}_{2}$ and $\mathrm{CaO}_{2}$ values showed a significant increase $(p<0.05)$ from T1 baseline moment. No significant changes were apparent after performing each maneuver in the time T3 of evaluation; however, there was a significant increase $(\mathrm{p}<0.05)$ in $\mathrm{PaO}_{2}$ of $13.5 \%$ in the time T4 after making the M2 maneuver. From which we conclude that the performance of alveolar recruitment maneuvers by artificial manual breathing units - AMBU are a safe alternative 
to assisted ventilation during alveolar recruitment maneuvers with PEEP lower than $20 \mathrm{cmH}_{2} \mathrm{O}$ in clinically healthy animals.

Key words: Anesthesia, atelectasis, gasometry, manual ventilation, oxygenation.

\section{INTRODUCCIÓN}

Bajo condiciones ideales cada unidad alveolar debería recibir la misma cantidad de aire inspirado y de sangre venosa, pero esta afirmación es solamente teórica, debido a la heterogenicidad alveolar, en diferentes áreas del pulmón. En la práctica clínica, la valoración del equilibrio entre ventilación y perfusión, se realiza mediante el estudio del intercambio gaseoso, en donde los valores de dióxido de carbono $\left(\mathrm{CO}_{2}\right)$ y de oxígeno $\left(\mathrm{O}_{2}\right)$ en sangre arterial son el reflejo de dicho equilibrio (Soro \& Belda, 2009; Corcoran, 2013) y deben ser tenidos en cuenta, durante el monitoreo ventilatorio.

La atelectasia pulmonar es considerada como la principal causa de hipoxemia intraoperatoria. Las áreas de atelectasia se localizan, fundamentalmente, en las zonas dependientes del pulmón y afectan según el procedimiento quirúrgico realizado, entre un 5 y $50 \%$ de este órgano (Martínez \& Cruz, 2008). El uso de fracciones inspiradas de oxígeno (FiO2), superiores al $80 \%$, favorecen la formación de atelectasias (Edmark et al. 2003), mientras que $\mathrm{FiO} 2$, inferiores al $60 \%$, han sido asociadas a una reducción preventiva de las mismas (Staffieri et al. 2010a). De acuerdo con Strandberg et al. (1986), las atelectasias generalmente desaparecen, tras las primeras $24 \mathrm{~h}$ del periodo postoperatorio, pero en otras ocasiones, pueden permanecer por varios días (Warner, 2000).

Diversas estrategias de distensión alveolar han sido desarrolladas para disminuir la presentación de atelectasias durante el periodo transanestésico, mediante la apertura de la mayor cantidad de unidades alveolares durante el mayor tiempo posible, a fin de mejorar el intercambio gaseoso (Perasso et al. 2006; Ibancovichi et al. 2009; Tusman \& Böhm, 2010; Ospina et al. 2014). Dentro de estas estrategias, se incluyen la presión positiva continua de la vía aérea (Rothen et al. 1993), posición prona (Gattinoni et al. 1993), suspiros intermitentes (Perasso et al. 2006), maniobra de capacidad vital (Martínez \& Cruz, 2008) y la empleada en el presente estudio, presión positiva al final de la espiración (PEEP) (Torrente, 2011; Canfrán et al. 2012). La mayoría de las anteriores estrategias son habitualmente realizadas, mediante el empleo de equipos de ventilación mecánica controlada.

La disponibilidad de equipos de ventilación mecánica controlada puede ser una limitante para la práctica de estrategias de distensión alveolar transanestésica en veterinaria, especialmente, en países en vía de desarrollo. Debido a lo anterior, el empleo de unidades de respiración artificial manual (AMBU), se constituye como una herramienta alternativa, que permite la aplicación de estrategias de distensión alveolar y soporte ventilatorio en la práctica médico veterinaria, en estos países (Maurya et al. 2008); sin embargo, del conocimiento de los autores, no se registran estudios publicados que demuestren cuál es el efecto del empleo del AMBU, como estrategia de distensión alveolar, sobre la dinámica de intercambio gaseoso en caninos. Tampoco existen evidencias que permitan dilucidar el efecto de estas maniobras, sobre el valor de gases sanguíneos, a las 24 horas de realizadas.

Debido a lo anterior, se planteó el presente estudio, con el objetivo de evaluar el efecto que tiene la realización de diversas maniobras cíclicas de reclutamiento alveolar, sobre el valor de gases sanguíneos, en perros anestesiados, empleando ventilación manual con $\mathrm{AMBU}$.

\section{MATERIALES Y MÉTODOS}

Este estudio fue avalado por el comité de bioética de la Universidad del Tolima y desarrollado bajo los lineamientos de las leyes 84 de 1989 y 1774 de 2016 de protección animal y desarrollado en la Clínica de Pequeños Animales de la Universidad del Tolima, localizada en Ibagué, Tolima - Colombia, a una altura sobre el nivel del mar de $1.527 \mathrm{msnm}$, una presión barométrica de $657 \mathrm{mmHg}$ y una temperatura promedio de $28,9^{\circ} \mathrm{C}$.

Veintiocho hembras caninas, con un peso comprendido entre 9,0 y $31,4 \mathrm{~kg}$, un rango de edades entre 17,5 y 25,7 meses, clínicamente sanas y sometidas a profilaxis dentales, bajo un protocolo de anestesia general inhalada, fueron incluidas en el presente estudio prospectivo. Los criterios de inclusión para los animales ingresados incluyeron: edad entre 1 y 8 años, peso entre 5 y $35 \mathrm{~kg}$, normalidad en los resultados del examen físico y paraclínico. Por su parte, los criterios de exclusión comprendieron: obesidad, braquicefalia, agresividad y nerviosismo evidente. Las condiciones generales en las que se desarrolló el estudio fueron: no se realizó medicación preanestésica, la anestesia general fue inducida con tiopental sódico a posología de $12 \mathrm{mg} / \mathrm{kg}$,IV y mantenida en ventilación espontánea con isoflurano en oxígeno al $100 \%$. La duración de la intervención (profilaxis dental) fue de 30 minutos, en todos los casos y los individuos fueron posicionados en decúbito lateral derecho, durante todo el tiempo anestésico.

Los animales incluidos en el estudio fueron asignados al azar, mediante un sistema de aleatorización en línea RANDOM. ORG, dentro de uno de los dos grupos experimentales a saber: El grupo uno (M1), reclutado mediante maniobra cíclica manual, a una PEEP progresiva de $10 \mathrm{cmH}_{2} \mathrm{O}$ y el grupo dos (M2), reclutado manualmente, con una PEEP progresiva de $20 \mathrm{cmH}_{2} \mathrm{O}$; el acondicionamiento hemodinámico y los tiem- 
pos de la maniobra, se encuentran referenciados en la figura 1. Las maniobras de reclutamiento alveolar fueron realizadas mediante el empleo de AMBU, a un volumen tidal de $10 \mathrm{~mL} /$ $\mathrm{kg}$, una presión de flujo inspiratorio de $10 \mathrm{cmH}_{2} \mathrm{O}$ y una relación inspiración:espiración (I:E) de 1:3. Los dispositivos AMBU fueron provistos de un manómetro integrado de presión inspiratoria y una válvula PEEP, para garantizar las condiciones ya mencionadas (Figura 2). Todas las maniobras de ventilación fueron desarrolladas por un mismo profesional entrenado y con experiencia en este tipo de maniobras.
Se obtuvieron muestras sanguíneas, mediante punción de la arteria femoral derecha o izquierda, en cuatro tiempos diferentes: T1 (basal en preanestesia), T2 (pre-reclutamiento), T3 (30 min post-maniobra) y T4 (24h post-maniobra). El T2 correspondió al momento en que los pacientes alcanzaron el plano anestésico III, para lo cual, fue empleado un resumen de los criterios de la clasificación de Guedel (Haskins, 2015): presencia de ventro rotación ocular, dilatación pupilar y tono mandibular disminuido; reflejos y respuestas fisiológicas a estímulos nociceptivos ausentes.

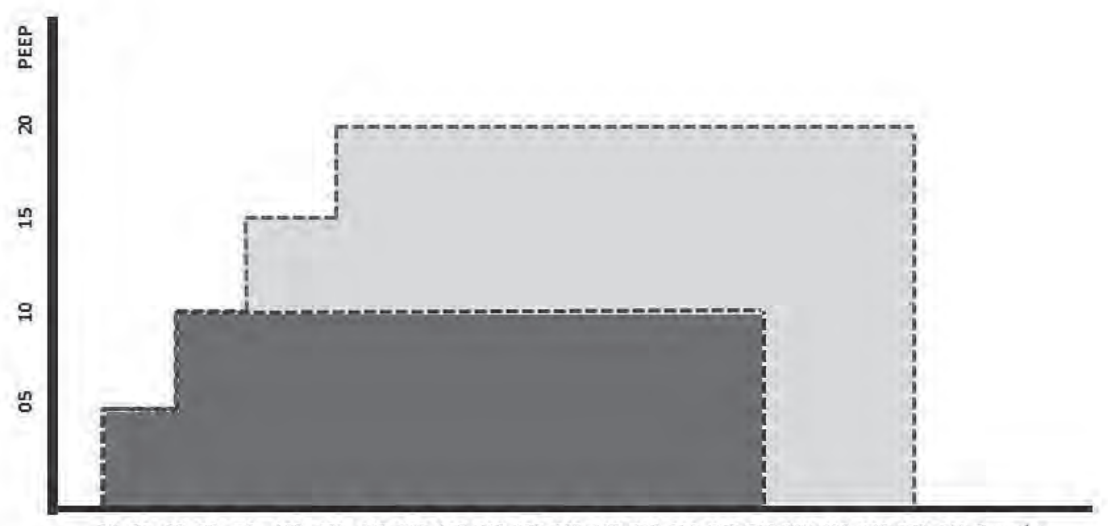

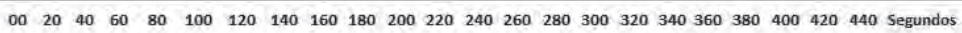

Figura 1. Relación entre la presión tele-espiratoria alcanzada y el tiempo de ejecución de la maniobra, para cada uno de los protocolos de reclutamiento, empleados en el estudio; grupo 1 (gris oscuro) y grupo 2 (gris claro). Los periodos de acondicionamiento hemodinámico, para los protocolos 1 y 2, se encuentran escalonados. El tiempo de reclutamiento para cada grupo fue de 300 segundos.

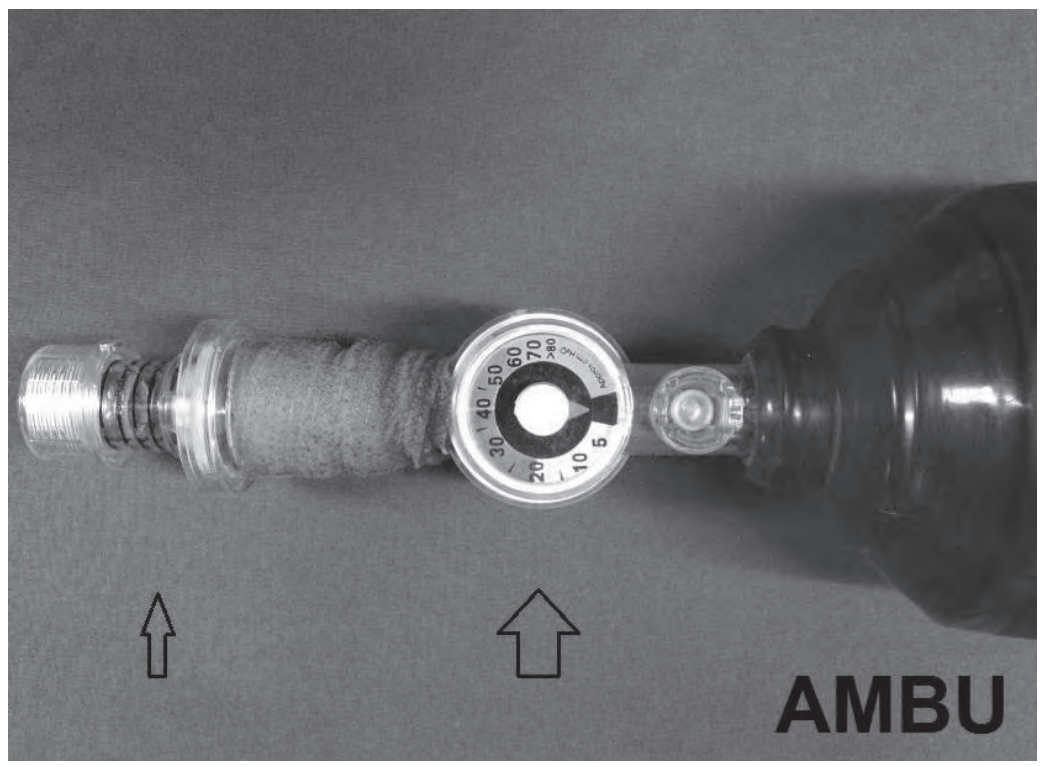

Figura 2. Unidad de respiración artificial manual -AMBU-, de sus siglas en idioma inglés artificial manual breathing unit. La flecha gruesa señala el manómetro integrado para control de presión inspiratoria; la flecha delgada señala la válvula ajustable de PEEP. 
Cada muestra sanguínea fue procesada inmediatamente, empleando un equipo de hemogasometría portátil (I-STAT 300 / CG4+, Abbott point of care, Ilinois). Las variables evaluadas fueron: presión arterial de oxígeno $\left(\mathrm{PaO}_{2}\right)$, presión arterial de dióxido de carbono $\left(\mathrm{PaCO}_{2}\right)$, dióxido de carbono total $\left(\mathrm{TCO}_{2}\right)$, relación presión arterial de oxígeno/fracción, inspirada de oxígeno $\left(\mathrm{PaO}_{2} / \mathrm{FiO}_{2}\right)$, saturación de oxígeno $\left(\mathrm{SpO}_{2}\right)$ y concentración arterial de oxígeno $\left(\mathrm{CaO}_{2}\right)$.

Los registros de la frecuencia cardiaca y ventilatoria, presión arterial no invasiva sistólica, diastólica y media, así como también de la temperatura rectal fueron evaluados en cada uno de cuatro tiempos experimentales estudiados.

Todas las variables numéricas fueron analizadas, a través de medidas descriptivas. El tiempo T1, se comparó con el tiempo T4 (animales no anestesiados) y el tiempo T2, con el T3 (animales anestesiados), lo anterior, con el fin de estudiar posibles diferencias entre grupos. De igual manera, las dos maniobras fueron contrastadas entre sí, en cada uno de los tiempos, T3 y T4. La distribución normal de los datos fue verificada por medio de la prueba de Shapiro Wilk. El test de análisis de varianza de una vía (ANOVA), para mediciones repetidas, fue empleado para definir la diferencia entre variables. Los análisis no paramétricos fueron realizados mediante la prueba U-Mann Whitney, como post hoc. Un valor de $p<0,05$ fue considerado como estadísticamente significativo. Los datos fueron analizados en el Software Graph Pad Prism 6.0, California, USA.

\section{RESULTADOS Y DISCUSIÓN}

El objetivo del presente estudio fue evaluar el efecto de dos maniobras manuales cíclicas de reclutamiento alveolar, mediante el incremento de la presión tele-espiratoria (PEEP), sobre seis parámetros de gases arteriales, en caninos anestesiados y ventilados, mediante el uso de AMBU. El actual estudio evidenció que el uso de las maniobras M1 y M2, empleadas en el presente trabajo, no generaron diferencias estadísticamente significativas en los parámetros sanguíneos evaluados en el tiempo T3, pero sí en el tiempo T4, para la maniobra M2, lo que hace suponer que el empleo de AMBU, a mayor presión y tiempo, podría tener un efecto sobre el valor de gases arteriales a las 24 horas de realizada cada maniobra de reclutamiento alveolar (MRA). Del conocimiento de los autores, este estudio es el primero en proveer información sobre los valores de referencia de los gases sanguíneos estudiados para cada uno de los tiempos evaluados, después de aplicar una MRA, mediante el empleo de AMBU; dichos resultados se muestra en la tabla 1 .

Los valores de frecuencia cardiaca, frecuencia ventilatoria, presión arterial y temperatura rectal, no evidenciaron cambios estadísticos significativos entre los grupos comparables T1 - T4 y T2 - T3 y estuvieron entre los rangos descritos para la especie.

Durante el tiempo T2, los valores de $\mathrm{PaO}_{2}, \mathrm{PaO}_{2} / \mathrm{FiO}_{2}, \mathrm{PCO}_{2}$ y $\mathrm{SpO}_{2}$ evidenciaron un incremento estadísticamente signifi-

Tabla 1. Valor de gases arteriales $( \pm \mathrm{SD}$ ) para cada uno de los protocolos evaluados, en los tiempos T1, T2, T3 (M1 y M2), T4 (M1 y M2), presión arterial de oxígeno $\left(\mathrm{PaO}_{2}\right)$, presión arterial de dióxido de carbono (PaCO2), dióxido de carbono total $\left(\mathrm{TCO}_{2}\right)$, relación presión arterial de oxígeno/fracción inspirada de oxígeno $\left(\mathrm{PaO}_{2} / \mathrm{FiO}_{2}\right)$, saturación de oxígeno $\left(\mathrm{SpO}_{2}\right)$ y concentración arterial de oxígeno $\left(\mathrm{CaO}_{2}\right)$.

\begin{tabular}{|l|c|c|c|c|c|c|}
\hline & & & \multicolumn{2}{|c|}{30 minutos } & \multicolumn{2}{c|}{ 24 horas } \\
\hline Variable & $\mathrm{T} 1$ & $\mathrm{~T} 2$ & $\mathrm{T3}(\mathrm{M} 1)$ & $\mathrm{T3}(\mathrm{M} 2)$ & T4 (M1) & T4 (M2) \\
\hline $\begin{array}{l}\mathrm{PaO}_{2} \\
(\mathrm{mmHg})\end{array}$ & $91,7( \pm 5,6)$ & $558,9( \pm 6,9)^{*}$ & $554,6( \pm 6,9)$ & $554,5( \pm 5,3)$ & $91,6( \pm 3,3)$ & $104,1( \pm 1,8)^{*}$ \\
\hline $\begin{array}{l}\mathrm{PCO}_{2} \\
(\mathbf{m m H g})\end{array}$ & $29,1( \pm 4,5)$ & $40,9( \pm 5,6)^{*}$ & $44,3( \pm 5,5)$ & $44,4( \pm 4,2)$ & $29,2( \pm 2,6)$ & $28,9( \pm 2,9)$ \\
\hline $\mathrm{TCO}_{2}(\mathbf{m m o l} / \mathrm{L})$ & $20,1( \pm 3,9)$ & $23,8( \pm 2,6)$ & $24,2( \pm 2,1)$ & $24,6( \pm 1,6)$ & $19,5( \pm 2,4)$ & $19,2( \pm 1,5)$ \\
\hline $\mathrm{PaO}_{2} / \mathrm{FiO}_{2}$ & $436,7( \pm 26,8)$ & $558,9( \pm 6,9)^{*}$ & $554,6( \pm 6,9)$ & $554,5( \pm 5,3)$ & $436,1( \pm 15,5)$ & $495,1( \pm 8,7)^{*}$ \\
\hline $\begin{array}{l}\mathrm{SpO}_{2} \\
(\%)\end{array}$ & $94,4( \pm 1,5)$ & $99,9( \pm 0,2)^{*}$ & $98,2( \pm 3,4)$ & $99,4( \pm 1,2)$ & $94,6( \pm 1,4)$ & $93,2( \pm 4,2)$ \\
\hline $\begin{array}{l}\mathrm{CaO}_{2} \\
(\mathrm{ml} / \mathrm{dl})\end{array}$ & $19,5( \pm 2,5)$ & $21,5( \pm 2,5)$ & $21,9( \pm 3,2)$ & $20,4( \pm 2,3)$ & $18,4( \pm 2,1)$ & $19,9( \pm 2,2)$ \\
\hline
\end{tabular}

* Significancia estadística $\mathrm{p}<0.05$. 
cativo ( $p<0,05)$, con respecto al T1 (Figura 3). Los demás parámetros de gases arteriales evaluados no mostraron diferencias estadísticas significativas en este tiempo. Se ha descrito que los fármacos barbitúricos ocasionan depresión respiratoria, sin cambios estadísticamente significativos sobre los parámetros de gases arteriales, tras la inducción anestésica (Robinson et al. 1986). Los incrementos presentados en los valores de gases arteriales, en el presente estudio, podrían ser asociados a la ventilación espontánea con isoflurano, vehiculizado en oxígeno al 100\%, empleada en los animales estudiados (Edmark et al. 2003; Soro \& Belda,
2009). En este sentido, se afirma que el uso de $\mathrm{FiO}_{2}$ superiores al $21 \%$ genera un incremento de la $\mathrm{PaO}_{2}$, en una relación cercana de 1:5 (Haskins, 2015), por lo tanto, la aplicación de ventilación con $\mathrm{FiO}_{2}$ al $100 \%$, llevaría a incrementos promedio de la $\mathrm{PaO}_{2}$ de $500 \mathrm{mmHg}$, valores que fueron observados en el monitoreo hemogasométrico, del presente estudio, en los tiempos T2 y T3; de igual manera y debido a que la $\mathrm{PaO}_{2} /$ $\mathrm{FiO}_{2}$, y la $\mathrm{SpO}_{2}$ son variables dependientes de la concentración de $\mathrm{O}_{2}$ en sangre, estos parámetros también evidencian un incremento en los tiempos relacionados.
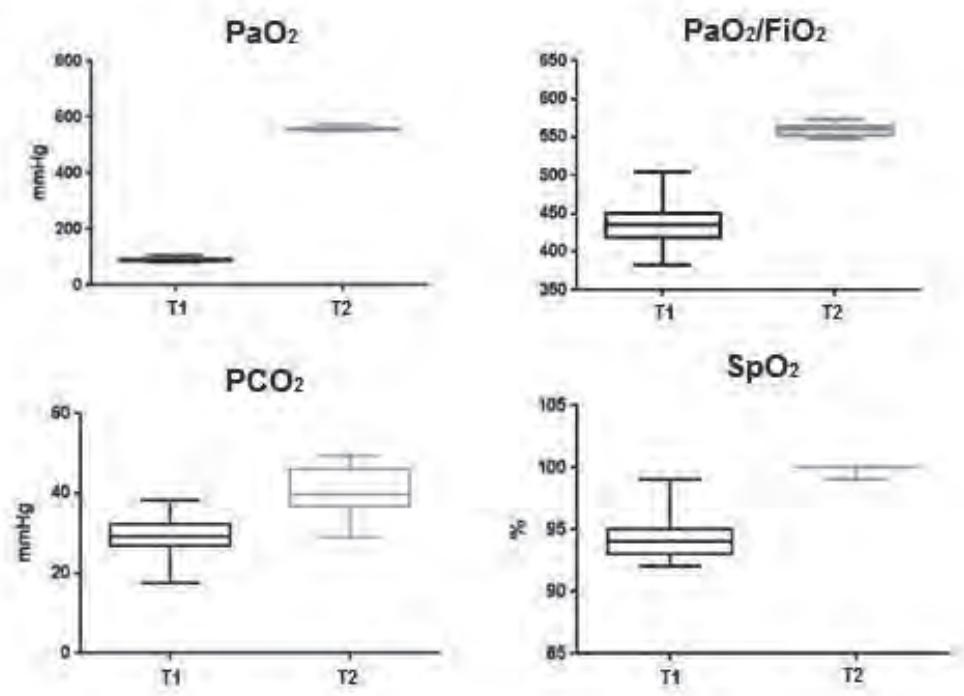

Figura 3. Comparación entre el valor de $\mathrm{PaO}_{2}, \mathrm{PaO}_{2} / \mathrm{FiO}_{2}, \mathrm{PCO}_{2}$ y $\mathrm{SpO}_{2}$ basal y el valor obtenido durante el T2 (pre-reclutamiento), con diferencia estadísticamente significativa $\mathrm{p}<0,05$.

La realización de las maniobras M1 y M2, empleadas en el presente estudio, no evidenciaron cambios estadísticamente significativos en el tiempo T3; sin embargo, durante el tiempo T4, se evidenció un incremento significativo del $13,5 \%$, tanto en la $\mathrm{PaO}_{2}$ como en la $\mathrm{PaO}_{2} / \mathrm{FiO}_{2}$, en la maniobra M2, con respecto al valor basal T1. Por su parte, estas mismas variables no mostraron cambios estadísticamente significativos entre la maniobra M1 y tiempo T1 (Figura 4); en las demás variables evaluadas no se evidenciaron diferencias estadísticas significativas, tras la realización de las maniobras. Los hallazgos obtenidos en el tiempo T3 contrastan con lo reportado por Trojik et al. (2012), quienes encontraron que diversas maniobras de reclutamiento alveolar en seres humanos generaron incrementos significativos en el valor de $\mathrm{PaO}_{2}, \mathrm{SaO}_{2}$ y $\mathrm{PaO}_{2} / \mathrm{FiO}_{2}$, a los 5 y 60 min de realizadas las maniobras; de igual manera, incrementos en los valores de $\mathrm{PaO}_{2}, \mathrm{PaO}_{2} / \mathrm{FiO}_{2}$ y $\mathrm{PaCO}_{2}$, a los 20 y 60 min posteriores a reclutamiento, mediante PEEP, han sido evidenciados en perros (Canfrán et al. 2012). Cabe resaltar que esos dos estudios fueron realizados empleando una $\mathrm{FiO}_{2}$ de 0,4 . La ausencia de cambios estadísticamente significativos en los parámetros evaluados para el T3, en el presente estudio, podrían estar relacionados con el empleo de una $\mathrm{FiO}_{2}$ alta, lo que generó incrementos sostenidos de la $\mathrm{PaO}_{2}$ y la $\mathrm{PaO}_{2} /$ $\mathrm{FiO}_{2}$, durante todo el evento anestésico, realizado en el presente estudio (Soro \& Belda, 2009).

Se afirma, que existe un incremento de la $\mathrm{PaO}_{2} / \mathrm{FiO}_{2}$, tras el aumento de la $\mathrm{FiO}_{2}$ en pacientes ventilados (Sánchez et al. 2011; Hernández \& Cerón, 2013). Cambios como este, soportan la relación existente entre la $\mathrm{PaO}_{2}$ y la $\mathrm{FiO}_{2}$, como una representación del estado de la mezcla venosa de gases sin efecto de cortocircuito pulmonar (Hernández \& Cerón, 2013); sin embargo, a pesar de mejorar la relación ventilación/perfusión $(\mathrm{V} / \mathrm{Q})$, el uso de $\mathrm{FiO}_{2}$ superiores al $80 \%$ podría conducir a la presentación de atelectasias por reabsorción (Edmark et al. 2003; Staffieri et al. 2010b; De Monte et al. 2013), incremento en el riesgo de intoxicación por $\mathrm{O}_{2}$, es- 

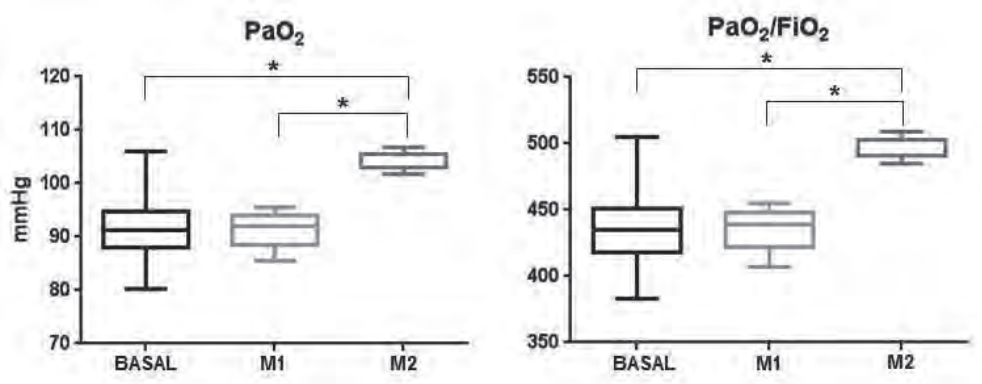

Figura 4. Diferencias estadísticamente significativas $\mathrm{p}<0,05$ entre los tiempos T1 y T4 (24 horas post-maniobra), para las variables $\mathrm{PaO}_{2}$ y $\mathrm{PaO}_{2} / \mathrm{FiO}_{2}$, en cada una de las Maniobras $\mathrm{M} 1$ y $\mathrm{M} 2$.

pecialmente, durante procesos anestésicos prolongados (Frávega-Pérez, 2011) y al subvalor en los parámetros de gases arteriales durante el monitoreo ventilatorio (Sánchez et al. 2011), como es el caso de la hipercapnia, aún en condiciones de hipoventilación, lo que puede conllevar a errores de interpretación, durante el monitoreo anestésico.

Algunos estudios han evidenciado un incremento en el valor de gases sanguíneos posteriores a MRA, durante periodos de tiempo inferiores a 60 min (Staffieri et al. 2010b; Canfrán et al. 2012; Trojik et al. 2012). Del conocimiento de los autores, tan solo un estudio de caso relacionó en seres humanos la utilización de MRA, de forma manual, con el empleo de AMBU, usando $\mathrm{FiO}_{2}$ del $100 \%$ y alcanzando un pico máximo de presión de $20 \mathrm{cmH}_{2} \mathrm{O}$, que permitió obtener un intercambio gaseoso óptimo, en términos de la $\mathrm{PaO}_{2} / \mathrm{FiO}_{2}$, tras su realización consecutiva por cinco días (Maa et al. 2005). La no evidencia de estos cambios a las 24 horas de realizadas las MRA, bajo los protocolos empleados en el presente estudio, resalta la importancia clínica del mismo, toda vez que en la práctica médico veterinaria en Colombia, el uso de AMBU es considerado como una herramienta de rutina para apoyar la ventilación transanestésica, en aquellos momentos en que la ventilación espontánea es insuficiente.

Un estudio realizado en pulmones de ratas demostró que el empleo de una maniobra de insuflación sostenida con $20 \mathrm{cmH}_{2} \mathrm{O}$ produce un reclutamiento alveolar de hasta $63,1 \pm 9 \%$, siendo la cantidad de alveolos reclutados, dependientes de esta presión alcanzada (Albert et al. 2009). Los resultados obtenidos en el presente estudio permiten inferir que, a las 24 horas de realizada una MRA, a una PEEP de $20 \mathrm{cmH}_{2} \mathrm{O}$, se generarán mayores alveolos funcionales en el tiempo, por lo tanto, el valor de gases sanguíneos asociados al oxígeno serán mayores, en contraste con una PEEP de $10 \mathrm{cmH}_{2} \mathrm{O}$.
La escasa disponibilidad de equipos anestésicos que permitan el suministro controlado de la mezcla aire y oxígeno a $\mathrm{FiO}_{2}$ inferiores al $40 \%$ es una limitante en la práctica médico veterinaria, en países en vías de desarrollo, lo que hace rutinario el uso de dispositivos de ventilación asistida con $\mathrm{FiO}_{2}$ del $100 \%$, situación que conlleva riesgos inherentes a su empleo. De igual manera, el uso de dispositivos manuales de ventilación tipo AMBU, por lo regular, carecen de mecanismos de control de flujo inspiratorio, lo que podría favorecer en casos de entrenamiento limitado del personal médico veterinario, la inducción de lesiones asociadas a barotrauma (Kim et al. 2010). El presente estudio no evidenció complicaciones clínicas asociadas a neumomediastino en los animales evaluados, durante las primeras 24 horas de realizadas las maniobras; se consideró que el riesgo de barotrauma fue minimizado, debido al entrenamiento previo del profesional, quien realizó la ventilación, así como también al control de la presión inspiratoria visualizada en la válvula de presión inspiratoria integrada en el AMBU. Por lo tanto, se puede afirmar que el empleo de AMBU con válvula de control de flujo inspiratorio y por personal previamente entrenado, siempre deberá ser prioritario al momento de realizar maniobras, a fin de minimizar riesgos inherentes al incremento de la presión inspiratoria.

Limitaciones del estudio: Tras la realización del presente estudio es importante considerar que no fue posible estimar el grado de atelectasia alveolar presentado en cada uno de los individuos evaluados, ni la minimización de la misma, tras el uso de las distintas maniobras. Estas condiciones podrían ser tenidas en cuenta para la realización de trabajos futuros, que incluyan el empleo de dispositivos tipo AMBU.

Los resultados obtenidos en el presente estudio permiten concluir, que el uso de maniobras cíclicas de reclutamiento alveolar, mediante el empleo de dispositivos tipo AMBU, en la práctica clínica de pequeños animales, puede ser una alternativa a la optimización de la dinámica ventilatoria, en 
pacientes con normalidad pulmonar, sometidos a anestesia general inhalada y un mejor efecto sobre la oxigenación se puede alcanzar, empleando un protocolo cíclico escalonado, hasta alcanzar una presión telespiratoria tope de $20 \mathrm{cmH}_{2} \mathrm{O}$ de PEEP; sin embargo, el uso de altas concentraciones de oxígeno en la vehiculización anestésica tiende a enmascarar algunos valores de gases sanguíneos evaluables durante el monitoreo anestésico, subestimando el diagnóstico de hipercapnia o de hipoxemia.

Conflictos de intereses: El manuscrito fue preparado y revisado con la participación de todos los autores, quienes declaramos que no existe conflicto de intereses que ponga en riesgo la validez de los resultados presentados. Financiación: Este estudio fue financiado por la Oficina de Investigaciones y Desarrollo Científico de la Universidad del Tolima.

\section{BIBLIOGRAFÍA}

1. ALBERT, S.P.; DIROCCO, J.; ALLEN, G.B.; BATES, J.H.; LAFOLLETTE, R.; KUBIAK, B.D.; FISCHER, J.; MARONEY, S.; NIEMAN, G.F. 2009. The role of time and pressure on alveolar recruitment. J. Appl. Physiol. (Estados Unidos). 106(3):757-765.

2. CANFRÁN, S.; GÓMEZ DE SEGURA, I.A.; CEDIEL, R.; GARCÍA-FERNÁNDEZ, J. 2012. Effects of a stepwise lung recruitment manoeuvre and positive endexpiratory pressure on lung compliance and arterial blood oxygenation in healthy dogs. Vet. J. (Inglaterra). 194(1):89-93.

3. CORCORAN, B. 2013. Fisiopatología respiratoria. En: Fuentes VL, Swift S (eds). Manual de medicina y cirugía cardiorrespiratorias en pequeños animales. Ed. S - Lexus (España).p.17-25.

4. DE MONTE, V.; GRASSO, S.; DE MARZO, C.; CROVACE, A.; STAFFIERI, F. 2013. Effects of reduction of inspired oxygen fraction or application of positive end-expiratory pressure after an alveolar recruitment maneuver on respiratory mechanics, gas exchange, and lung aeration in dogs during anesthesia and neuromuscular blockade. Am. J. Vet. Res. (Estados Unidos). 74(1):25-33.

5. EDMARK, L.; KOSTOVA-AHERDAN, K.; ENLUND, M.; HEDENSTIERNA, G. 2003. Optimal oxygen concentration during induction of general anesthesia. Anesthesiology. (Estados Unidos). 98(1):28-33.

6. FRÁVEGA-FLÓREZ, R. 2011. Revisión: Toxicidad pulmonar por oxígeno. Hospitales Veterinarios (Chile). 3(1):31.34.
7. GATTINONI, L.; D’ANDREA, L.; PELOSI, P.; VITALE, G.; PESENTI, A.; FUMAGALLI, R. 1993. Regional effects and mechanism of positive end-expiratory pressure in early adult respiratory distress syndrome. JAMA. (Estados Unidos). 269(6):2122-2127.

8. HASKINS, S.C. 2015. Monitoring Anesthetized Patients. En: Grimm KA, Lamont LA, Tranquilli WJ, Greene SA, Robertson SA (eds). Veterinary Anesthesia and Analgesia The Fifth Edition of Lumb and Jones. Ed. Wliey-Blackwell (lowa). p.86-113.

9. HERNÁNDEZ G.; CERÓN U. 2013. Influencia de la PEEP en la correlación entre la $\mathrm{DA}-\mathrm{aO} 2$ y la $\mathrm{PaO} 2 / \mathrm{FiO} 2$. Revista de la Asociación Mexicana de medicina crítica y terapia intensiva. (México). 27(4):226-230.

10. IBANCOVICHI, J.A.; MORAN R., A.; ARCIQUE, M.; VICTORIA, J.M. 2009. Introducción a la ventilación mecánica en la unidad de cuidados críticos. Rev. AMMVEPE. (México). 20(5):128-132.

11. KIM, J.B.; JUNG, H.J.; LEE, J.M.; IM, S.K.; KIM, D.J. 2010. Barotrauma developed during intra-hospital transfer: A case report. Korean J Anesthesiol. (Korea). 59(suppl):s218-s221.

12. MAA, S.H.; HUNG, T.J.; HSU, K.H.; HSIEH, Y.I.; WANG, K.Y.; WANG, C.H.; LIN, H.C. 2005. Manual hyperinflation improves alveolar recruitment in difficult-to-wean patients. CHEST. (Estados Unidos). 128(4):2714-2721.

13. MARTÍNEZ, G.; CRUZ, P. 2008. Atelectasias en anestesia general y estrategias de reclutamiento alveolar. Rev. Esp. Anestesiol. Reanim. 55(1):493-503.

14. MAURYA, P.K.; KALITA, J.; PALIWAL, V.K.; MISRA, U.K. 2008. Manual AMBU ventilation is still relevant in developing countries. QJM. (Inglaterra). 101(12):990991.doi: 10.1093/qjmed/hcn113.

15. OSPINA, D.A.; BURITICÁ, E.F.; ECHEVERRY, D.F.; VILLANUEVA-RODRÍGUEZ, C.L.; RONDÓN-BARRAGÁN, I.S. 2014. Maniobras de reclutamiento alveolar en el control de la atelectasia pulmonar: revisión de literatura. Veterinaria y Zootecnia. (Colombia). 8(1):17-34.

16. PERASSO, O.; CAPURRO, J.; SANZ, R.; GÓMEZ, L. 2006. Maniobras de reclutamiento alveolar. Rev. Argent. Anestesiol. 64(5):201-215. 
17. ROBINSON, E.P.; SAMS, R.A.; MUIR, W.W. 1986. Barbiturate anesthesia in greyhound and mixed-breed dogs: comparative cardiopulmonary effects, anesthetic effects, and recovery rates. Am. J. Vet. Res. (Estados Unidos). 47(10):2105-2112.

18. ROTHEN, H.U.; SPORRE, B.; ENGBERG, G.; WEGENIUS G.; HEDENSTIERNA, G. 1993. Reexpansion of atelectasis during general anaesthesia: a computed tomography study. Br. J. Anaesth. (Inglaterra). 71(6):788-795.

19. SÁNCHEZ, M.; QUINTANA, M.; PALACIOS, D.; HORTIGÜELA, V.; MARCO, C.; GARCÍA, J.; CANABAL, A.; PÉREZ, J.M.; VELASCO, A.; ARRESE, M.A. 2011. Relación entre el gradiente alveolo-arterial de oxígeno y la $\mathrm{PaO} 2 / \mathrm{FiO} 2$ introduciendo la PEEP en el modelo. Medicina intensiva. (España). 36(5):329-334.

20. SORO, M.; BELDA, F.J. 2009. Intercambio de gases. Relación ventilación - perfusión, oxigenación y eliminación de $\mathrm{CO}_{2}$. En: Belda FJ, Lloréns J. Ventilación mecánica en anestesia y cuidados críticos. Arán ediciones S.L. (España). p.107-139.

21. STAFFIERI, F.; DE MONTE, V.; DE MARZO, C.; GRASSO, S.; CROVACE, A. 2010a. Effects of two fractions of inspired oxygen on lung aeration and gas exchange in cats under inhalant anaesthesia. Vet. Anaesth. Analg. (Inglaterra). 37(6):483-490.

22. STAFFIERI, F.; DE MONTE, V.; DE MARZO, C.; SCRASCIA, F.; CROVACE, A. 2010b. Alveolar recruiting maneuver in dogs under general anesthesia: effects on alveolar ventilation, gas exchange, and respiratory mechanics. Vet. Res. Commun. (Holanda). 34(1):S131-S134.

23. STRANDBERG, A.; TOKICS, L.; BRISMAR, B.; LUNDQUIST, H.; HEDENSTIERNA, G. 1986. Atelectasis during anaesthesia and in the postoperative period. Acta. Anaesthesiol. Scand. (Inglaterra). 30(2):154158.

24. TORRENTE, C. 2011. Ventilación mecánica en el pulmón patológico. Clin. Vet. Peq. Anim. (España). 31(2):77-84.

25. TROJIK, T.; SHOSHOLCHEVA, M.; RADULOVSKACHABUKOVSKA, J.; LOVACH-CHEPUJNOSKA, $M$. 2012. Evaluation of effects of repetitive recruitment maneuvers. Acta. Inform. Med. (Bosnia y Herzegovina). 20(2):85-89.

26. TUSMAN, G.; BÖHM, S. 2010. Prevention and reversal of lung collapse during the intra-operative period. Best. Pract. Res. Clin. Anaesthesiol. (Holanda). 24(1):183197.

27. WARNER, D.O. 2000. Preventing postoperative pulmonary complications: The role of the anesthesiologist. Anesthesiology. (Estados Unidos). 92(5):1467-1472.

Recibido: Septiembre 23 de 2015

Aceptado: Mayo 17 de 2016

\section{Cómo Citar:}

Buriticá Gaviria, E.; Echeverry Bonilla, D.; Ospina Argüelles, D.: Efecto de dos maniobras manuales cíclicas de reclutamiento alveolar sobre gases sanguíneos en perros anestesiados. Rev. U.D.C.A Act. \& Div. Cient. 19(1): $161-168$. 